\section{ACCESO ABIERTO: \\ POSIBILIDADES Y RETOS EPISTEMOLÓGICOS PARA LAS PUBLICACIONES CIENTÍFICAS EN LA RED1}

\author{
Roberto Feltrero \\ Departamento de Lógica, Historia y Filosofía de la Ciencia \\ Facultad de Filosofía, UNED \\ C/ Senda del Rey, 7, 28040 Madrid, España \\ rfeltrero@gmail.com
}

\section{OPEN ACCESS; \\ POSSIBILITIES AND \\ EPISTEMOLOGICAL \\ CHALLENGES FOR SCIENTIFIC PUBLICATIONS ON THE WEB}

ABSTRACT: The different proposals for Open Access to scientific publishing on the Internet offer undeniable advantages for the scientific practices of communication, publishing, dissemination and validation of scientific results. However, neither these practical advantages, nor the economical and moral reasons that are usually alleged to justify the Open Access movement, seem to be enough to strengthen and extend this system to the whole scientific community. Those reasons might be supplemented, from a philosophical perspective, by contemplating the epistemological potential of Open Access and its practical possibilities for an Open Peer-Review system. This study will help us consider which possibilities can be explored and exploited within the Open Access Movement in order to unify proposals and methodologies and, by means of these, provide a valuable epistemological alternative to the current commercial toll-access system.

KEY WORDS: Open access, scientific publishing, social epistemolo$g y$, scientific practices, open peer-review.
RESUMEN: Las propuestas de acceso abierto a las publicaciones cientificas en Internet presentan innegables ventajas para las labores de comunicación, publicación, difusión y contrastación de los resultados científicos. Sin embargo, ni estas ventajas prácticas, ni las razones económicas y morales que habitualmente se esgrimen para justificar la propuesta del acceso abierto, parecen ser suficientes para afianzar y extender este sistema a toda la comunidad científica. Dichas razones pueden complementarse, desde la filosofía, con una revisión de las posibilidades epistemológicas del acceso abierto y sus propuestas sobre los sistemas abiertos de revisión de los artículos científicos. Este estudio servirá para comprender las posibilidades que el movimiento del acceso abierto puede explorar y explotar para unificar sus propuestas y sus metodologías de forma que pueda constituirse en una alternativa epistemológicamente más valiosa que el actual sistema de publicación comercial de acceso restringido.

PALABRAS CLAVE: Acceso abierto, publicaciones científicas, epistemologia social, prácticas científicas, revisión por los iguales, revisión abierta.






\section{INTRODUCCIÓN}

Las innegables ventajas que el modelo de acceso abierto a las publicaciones científicas en Internet presenta para las labores de comunicación, publicación, difusión y contrastación de los resultados científicos han resultado, de facto, insuficientes para que este modelo sea adoptado por toda la comunidad científica. A pesar de más de quince años de discusión intelectual², las razones epistemológicas, económicas y morales esgrimidas por los defensores del acceso abierto -razones generalmente provenientes del más elemental sentido común- han chocado una y otra vez con los argumentos que sustentan los sistemas de publicación comerciales implantados en la comunidad científica. Aunque, cada vez más, estas propuestas se ven reforzadas por las apuestas de instituciones académicas e, incluso, por organismos políticos ${ }^{3}$ que recomiendan y fomentan el acceso abierto a los resultados científicos, el sistema de reconocimiento y recompensa del trabajo científico sigue basándose en la publicación de los resultados científicos en las revistas indexadas, la inmensa mayoría de las cuales son revistas comerciales de acceso restringido 4 . No es extraño, por ello, que los investigadores sigan concentrando sus esfuerzos en este sistema de publicación y revisión mediadas por editores comerciales.

La inercia del citado sistema parece difícil de vencer a la hora de permitir una mayor implantación de las publicaciones de acceso abierto. Pero quizá esta afirmación no sea del todo exacta. En realidad, ya podemos encontrar un creciente número de artículos científicos a los que se puede acceder libremente en la Red en las páginas web personales de los científicos, en los distintos repositorios institucionales o en el creciente número de revistas científicas de acceso abierto. Sin embargo, estos artículos no contribuyen, o lo están haciendo muy lentamente, a un cambio en el modelo de publicación y revisión. Una de las razones para ello es que las diversas propuestas sobre el acceso abierto difieren sustancialmente en cuanto a su compromiso con modelos de revisión y contrastación de los resultados de los artículos científicos. Puesto que el argumento principal para sostener el actual sistema de publicaciones con editores comerciales es que éstos se encargan de organizar y coordinar los mecanismos que garantizan la fiabilidad y relevancia de lo que se publica, la propuesta del acceso abierto debe trabajar en la dirección de ofrecer una alternativa unificada a este sistema. Para ello, es necesario articular sus posibilidades en un modelo capaz de ofrecer un sistema completo de creación, revisión, publicación y difusión de la investigación científica que sea más fiable que el actual. El análisis detallado de estas prácticas científicas desde un punto de vista epistemológico puede permitir encontrar las claves y criterios para el diseño de tal sistema $y$, a la vez, verificar sus posibles ventajas epistemológicas frente al sistema actual.

La tesis principal de este artículo es, por tanto, que la desconfianza de los científicos y sus instituciones evaluadoras ante el movimiento del acceso abierto se puede salvar proponiendo un marco epistemológico común que evite la disparidad en las diversas propuestas de acceso abierto, muchas de las cuales no explotan todas las posibilidades epistemológicas que el acceso abierto brinda a los científicos. Para elaborar esta propuesta, y después de introducir al lector en las características básicas del movimiento del acceso abierto, se estudiarán los diversos modelos de publicación en acceso abierto desde un punto de vista epistemológico. Finalmente, se llevará a cabo un estudio más detallado de las posibilidades del modelo de revisión abierta en la Red (Open Peer-Review) como base para ese posible nuevo sistema global que pueda constituirse en una alternativa epistemológicamente valiosa.

\section{TeCnologías digitales, publicaciones Y ACCESO ABIERTO}

Las publicaciones científicas en formato digital, tanto las comerciales como las de acceso abierto, sacan provecho de las caracteristicas de las tecnologias computacionales ${ }^{5}$ para ofrecer nuevos servicios y posibilidades con innegables ventajas epistemológicas y, en algunos casos, sociales. La influencia de los computadores y de Internet en las actividades diarias de los investigadores científicos no se limita a las posibilidades de difusión de sus trabajos a través de la Red, sino que está induciendo un gran número de cambios en las prácticas científicas. Cambios que apuntan, aparte de otras consideraciones metodológicas (Feltrero, 2007), a una mayor distribución, cognitiva, tecnológica y social de la labor de los científicos. En el caso de las prácticas relacionadas con las publicaciones científicas, dichas tecnologías hacen posible la mecanización de las labores de búsqueda, catalogación, almacenaje, recuperación, copia 
e impresión de los artículos científicos. La aparición de sofisticados portales de Internet con motores de búsqueda especializados ${ }^{6}$ supone un cambio importante en las prácticas de investigación bibliográfica que ahora se realizan siempre mediante las herramientas computacionales de la Red y, en la mayoría de los casos, directamente en los contenidos disponibles en ella.

Pero las posibilidades epistemológicas de este entorno tecnológico no acaban en las búsquedas bibliográficas. Si las citas y referencias bibliográficas son un modo de aumentar el conocimiento que se hace accesible a través de un texto académico, ahora esa accesibilidad puede ser, incluso, inmediata. Por ejemplo, los enlaces hipertextuales funcionan como referencias o notas al pie en las que se puede ofrecer sin dificultad un enlace directo al artículo o la información citada. Siempre que el artículo o información estén disponibles en la Red, el conocimiento ya no es sólo citado, sino que es presentado de manera inmediata. Por ello, es más sencillo poner a disposición del lector las evidencias relevantes que justifican las afirmaciones de un texto científico. Desde el punto de vista epistemológico, este tipo de comunicación ofrece grandes posibilidades para, por un lado, aumentar el conocimiento que proporciona un artículo científico, facilitando el acceso a todo el conocimiento relativo al mismo y, por otro, asegurar la fiabilidad y veracidad del conocimiento presentado puesto que el acceso inmediato facilita y acelera las labores de confirmación (Goldman, 1999, 166-170).

Estas posibilidades se explotan ya en las revistas digitales en acceso abierto y también en las revistas comerciales. Lógicamente, en el caso de las revistas comerciales, para trabajar con sus motores de búsqueda y tener acceso a los enlaces proporcionados es necesario pagar las correspondientes cuotas de suscripción. Debido a estas ventajas tecnológicas, los editores comerciales han fijado, desde Por un lado, para proporcionar los nuevos servicios posibles gracias a las tecnologías computacionales a la comunidad investigadora. Por otro lado, porque, frente a la edición en papel, las revistas en formato electrónico ofrecen a los editores una reducción de costes más que significativa en los gastos de distribución. Aunque es cierto que el diseño y la puesta en funcionamiento de las plataformas digitales para la catalogación, búsqueda y recuperación de los archivos suponen fuertes inversiones, dichas inversiones hace ya tiempo, sus objetivos en el nuevo entorno digital. resultan pequeñas a medio y largo plazo frente a los gastos fijos de distribución de los ejemplares en papel. El gasto inicial se acaba amortizando por dos razones. Por un lado, una vez puesta en funcionamiento, los gastos de mantenimiento de la plataforma son muy pequeños. Por otro, dichas plataformas son reutilizadas una y otra vez para diferentes revistas de un mismo grupo editorial. El coste inicial de la digitalización, por tanto, debe ser calculado en función del tiempo de uso y la versatilidad de las tecnologías usadas. De hecho, estos gastos se minimizan gracias a las nuevas tecnologías cuando se implementan sistemas que hacen posible que los propios científicos se encarguen de muchas de estas labores ${ }^{7}$, por lo que empieza a ponerse en cuestión la necesidad de algunas labores editoriales que ahora se puede automatizar con los computadores.

Las ventajas tecnológicas y económicas, es decir, las posibilidades que tenían los científicos para editar y distribuir directamente y sin demoras sus trabajos mediante las mismas herramientas computacionales que ya tenian a su disposición en sus centros universitarios para otras labores, fueron el detonante de los primeros ensayos de una comunicación científica en acceso abierto mediante listas de correo, repositorios de archivos digitales, etc. Todos estos nuevos modos de comunicación científica, previos incluso al actual movimiento de acceso abierto cuya motivación es ofrecer revistas de investigación alternativas a las excesivamente caras revistas comerciales ${ }^{8}$, motivan un movimiento más amplio que trata de aprovechar todas las posibilidades de estas tecnologias para complementar mejorar $y$, finalmente, sustituir el sistema de publicación tradicional (Stallman, 2004, 121-125).

Pero, más allá de los aspectos prácticos y económicos, los argumentos más interesantes para justificar el acceso abierto pertenecen al ámbito moral y epistemológico. Las justificaciones morales provienen de los principios de justicia y equidad que, en una sociedad en la que la producción de conocimiento ha adquirido tanta importancia deben extenderse al acceso igualitario a la información y el conocimiento (Lipinski y Britz, 2000). Si tanto los aspectos productivos como el bienestar de una sociedad dependen en gran medida de su capacidad de producción de conocimiento científico y tecnológico, es justo tratar de eliminar todas las barreras para que los más desfavorecidos puedan acceder a las herramientas de producción de ese conocimiento. Los recursos electrónicos para la comunicación

ARBOR CLXXXV 737 mayo-junio [2009] 597-609 ISSN: 0210-1963

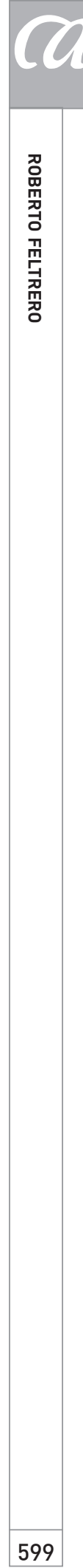

doi: 1040/arbor.2009.185 n.737 
pueden llegar a posibilitar el acceso universal a la literatura científica. Por ello, sería injusto no aprovechar estas posibilidades y seguir imponiendo barreras comerciales 0 económicas para el acceso a este tipo de conocimiento.

Desde el punto de vista de las comunidades científicas, la inmediatez a la hora de difundir los resultados investigadores y las oportunidades para una mayor colaboración entre un mayor número de expertos puede suponer mejorar y acelerar el proceso de solución de problemas científicos (Dominy y Bhatt, 2006). Ventajas que se ven mermadas cuando las revistas científicas tienen acceso restringido puesto que no todas las universidades o centros de investigación pueden costearse los altos precios de las suscripciones ${ }^{9}$.

Apoyándose en estos argumentos, han surgidos en los últimos años numerosas iniciativas para promocionar el acceso abierto ${ }^{10}$ a las publicaciones científicas cuyo denominador común es garantizar la libre disponibilidad de los textos académicos y científicos a través de Internet para su uso con fines investigadores o escolares. Con ello se trata de evitar cualesquiera de las trabas que la regularización comercial de los derechos sobre las publicaciones científicas pueda ocasionar a la creación de un sistema de publicación científica abierto y justo.

Estos argumentos también se han visto reforzados por argumentos prácticos en función de los parámetros e indicadores institucionales. Varios estudios muestran que la publicación en abierto de versiones digitales de los artículos científicos incrementa la visibilidad y el número de citas recibidas (Harnad y Brody, 2004; Pringle, 2004). De ahí que muchas revistas comerciales estén facilitando a los científicos la publicación en Internet de versiones previas (pre-prints) de sus artículos (Willinsky, 2006, 4-5).

Frente a toda esta panoplia de argumentos y datos favorables al nuevo modelo de acceso abierto, las revistas tradicionales, y muchos científicos, basan su defensa del sistema de acceso restringido en argumentos relacionados con su papel en el proceso de revisión y selección del conocimiento que publican. La revisión por los iguales (Peer Review), la selección y, en su caso, la corrección, que se lleva a cabo en el proceso de publicación asegura la calidad, la fiabilidad y la relevancia de lo que se publica. Frente a este argumento, el movimiento del acceso abierto no tiene una postura unitaria, como se explicará en el siguiente apartado.

Pero para mejor abordar esa cuestión, es necesario estudiar cuáles son las novedades proporcionadas por la digitalización y libre difusión de la investigación científica a través de Internet que pueden ayudar a mejorar la calidad, fiabilidad y relevancia de los artículos científicos. Las ventajas tecnológicas del acceso abierto no se reducen simplemente a la publicación y difusión en abierto de los artículos científicos en Internet. Si tomamos en cuenta las posibilidades multimedia de Internet, ahora los artículos pueden ofrecer, además del propio texto, materiales diversos tales como gráficos, simulaciones computacionales, bases de datos, etc. Es decir, la comunicación pública de los resultados científicos puede extenderse también a los recursos computacionales que los científicos usan para sus investigaciones, de manera que se facilita el estudio de todos los aspectos de la investigación e, incluso, se proporcionan todos los datos necesarios para la reproducción de sus experimentos.

Si los investigadores publican todos los datos y experimentos digitalizados relacionados con sus investigaciones, Internet puede convertirse en algo más que un simple almacén universal de información para pasar a ser un verdadero centro de investigación virtualizado. Todos estos datos podrian integrarse con el resto de las prácticas y objetos de la investigación electrónica (e-research) para hacer compatibles las mejoras del uso de computadores en los laboratorios, con las mejoras en los procesos de comunicación de la ciencia, incrementando ostensiblemente su transparencia.

Todas estas posibilidades tecnológicas deben ser objeto de una reflexión epistemológica que ayude a dilucidar en qué medida facilitan la creación de conocimiento fiable. Sin duda, el enriquecimiento de la comunicación científica y su transparencia mejorarian la fiabilidad de los artículos científicos. El proceso de contrastación de los resultados científicos por otros científicos sería mucho más sencillo -en muchos casos casi automático- y se reducirian significativamente, sino por completo, las posibilidades de fraude.

Si nos centramos en las posibilidades tecnológicas para las labores de revisión de las publicaciones científicas, 
hay que destacar que Internet proporciona un espacio de publicación y almacenamiento virtualmente irrestricto. Ello hace posible dar cabida a todo tipo de sistemas que permiten publicar, de manera síncrona y asíncrona, todas las comunicaciones, revisiones, comentarios, actualizaciones y versiones actualizadas de los resultados científicos. Los artículos podrían ganar así en relevancia, tanto por el enriquecimiento de los mismos con las discusiones durante el proceso de revisión como, y de manera más significativa, con la posibilidad de publicar sucesivas versiones mejoradas de los mismos. Si estas revisiones y mejoras fueran contabilizadas en los sistemas de reconocimiento de la actividad investigadora, los científicos podrían dedicar más tiempo y esfuerzo a esas labores haciendo que ese artículo, así como las discusiones a él asociadas, ganasen en relevancia. Desgraciadamente, hoy en día esos sistemas de reconocimiento, así como la exigencia de las revistas de publicar artículos completamente originales, hace que los científicos reduzcan la relevancia de sus artículos publicando versiones más o menos novedosas de las mismas ideas en diferentes trabajos o publicaciones.

Finalmente, aunque no es fácil establecer una relación causal directa, es fácil aventurar que un sistema de publicación tan completo y exhaustivo podría mejorar diferentes aspectos de la calidad de los artículos científicos. Fundamentalmente, gracias a las exigencias de precisión de los datos que implica una metodología de publicación transparente, así como al compromiso con las discusiones públicas que exigiría un sistema de comunicación abierta.

Todas estas herramientas de comunicación global entre los científicos en los distintos niveles y procesos, pueden mejorar la producción colaborativa de conocimiento, cuantitativa y cualitativamente. Al menos, en principio, parece claro que suponen afianzar y poner verdaderamente en acción principios epistemológicos constitutivos de la investigación científica, como son la transparencia y la publicidad, así como los mecanismos de reconocimiento y relevancia por medio del consenso de la comunidad científica. La iniciativa de la revisión abierta (Open Peer Review) se basa en estas últimas posibilidades tecnológicas para mejorar los procesos de discusión. Es preciso, por tanto, estudiar si el acceso abierto puede integrar y sacar partido de estas posibilidades para mejorar los sistemas de revisión y confirmación de los resultados científicos.

\section{ACCESO ABIERTO Y EPISTEMOLOGÍA}

La normatividad epistémica de la ciencia depende de la colaboración crítica y el consenso en su contexto social (Goldman, 2002; Longino, 1990). Las prácticas conducentes a ese consenso se han demostrado útiles para la producción, difusión y adquisición de conocimiento acreditado y confiable. Sin duda, la publicación de los resultados científicos es la práctica más extendida para lograr este contexto. Práctica, por otro lado, aceptada y apoyada sin fisuras desde las versiones más clásicas a las más postmodernas de los estudios epistemológicos. La evaluación epistemológica del movimiento del acceso abierto debería comenzar, por tanto, por la evaluación de sus posibilidades a la hora de asegurar la difusión, la acreditación y confiabilidad del conocimiento. El sistema de revisión previa de los artículos por los iguales (peer review), ha sido, hasta hoy, el principal garante de esas cualidades epistemológicas, por lo que es preciso estudiar estos sistemas en el movimiento del acceso abierto.

Pero, como ya se ha destacado anteriormente, no existe una propuesta uniforme en este sentido. Todo lo contrario, es posible encontrar diversas metodologías para la publicación de los trabajos científicos de manera que sean libremente accesibles a través de Internet. Diversas metodologías que también difieren en cuanto al sistema de revisión de los artículos. Es posible encontrar modelos de publicación en los que existe una revisión por otros científicos similar a la de las revistas tradicionales, otros en los que no hay revisión previa $y$, finalmente, modelos de autoarchivado de los borradores que, en algunos casos, experimentan con nuevas formas de revisión paralela. A continuación se estudiarán y clasificarán las propuestas del acceso abierto desde este punto de vista.

La gran mayoría de las revistas de acceso abierto ofrecen las mismas metodologías de revisión editorial previa que las revistas comerciales ${ }^{11}$. Desde este punto de vista, las revistas de acceso abierto pueden "competir" en igualdad de condiciones con las revistas tradicionales puesto que ofrecen las mismas garantías en función de los especialistas dedicados al trabajo de revisión y selección de los artículos. Sin embargo, la organización del sistema de revisión puede requerir cierto esfuerzo e inversión, lo que se usa como argumento para justificar que algunas revistas de acceso abierto estén empezando a cobrar a los autores 
por publicar en ellas ${ }^{12}$. En este sentido, estas revistas de acceso abierto coinciden en los argumentos económicos de las revistas de acceso comercial. Por ejemplo, algunas revistas comerciales tradicionales empiezan a ofrecer a los científicos la posibilidad de ofrecer los artículos que publiquen en ellas en régimen de acceso abierto, siempre que dichos autores paguen una compensación económica a la propia revista. Cantidad que, como en el caso de algunas revistas de acceso abierto, se justifica en función del coste de los servicios de revisión y publicación.

Las iniciativas que incluyen el pago del autor para garantizar el acceso abierto a sus documentos pueden resultar contradictorias con los principios del acceso abierto pues ahora el acceso al conocimiento puede restringirse en función de las posibilidades económicas de los autores o las instituciones que financian sus actividades investigadoras. La discusión que se genera alrededor de este tema es si la actividad de la revisión de los textos debe ser remunerada o no. Aquí deberiamos distinguir entre la revisión académica (peer-review) que llevan a cabo los investigadores expertos y la revisión editorial para la mejora del estilo de los documentos. La primera, muy rara vez es remunerada, $\mathrm{ni}$ en las revistas comerciales ni en las revistas de acceso abierto, por lo que difícilmente puede servir de excusa para exigir el pago a los autores.

Otro modelo usado por las revistas de acceso abierto $y_{\text {, }}$ últimamente, también en boga en las revistas comerciales, es el de los comentarios publicados al lado de los artículos. En este caso, se aprovechan las posibilidades tecnológicas de edición en línea para que, o bien expertos autorizados por la revista, o bien cualquier interesado, puedan añadir, desde su propio computador, los comentarios o críticas que le suscite la lectura. Es una interesante forma de hacer explícito el sistema de revisión posterior de los artículos por toda la comunidad científica, haciendo accesible a todos el conocimiento adicional que esos comentarios o críticas aportan. Este tipo de práctica se suele denominar de comentarios abiertos (open commentary), práctica que, para algunos autores, tiene funciones y objetivos epistemológicos muy diferentes frente a la revisión por los iguales antes de la publicación (Harnad, 1998).

Finalmente, existen dos modelos de publicación en acceso abierto que no implican ninguna metodología específica para la revisión previa de los artículos. Se trata de la publicación de artículos en acceso abierto en las páginas personales de los científicos y el modelo de organización de esos artículos y materiales en los repositorios (archivos electrónicos) institucionales. La mayoría de los científicos se han planteado por primera vez la cuestión del acceso abierto a la hora de publicar o enlazar sus trabajos y artículos en sus páginas web personales. Dichos artículos son, muchas veces, las copias digitalizadas mediante un escáner de las ediciones impresas de sus trabajos. En realidad, esta práctica requiere el permiso de las editoriales en las que se publicó el artículo por lo que, en algunos casos, puede producir lagunas legales en la medida en que no se especifica qué es lo que nos está permitido hacer con esos textos. Por otro lado, muchos autores han optado por la publicación de versiones electrónicas de los borradores de sus documentos en sus propias páginas web ${ }^{13}$. Práctica que, como ya se apuntó anteriormente, está permitida por un número cada vez mayor de revistas y se reconoce explícitamente en los contratos de cesión del copyright. Pero en la mayoría de las ocasiones, en estas prácticas no hay un compromiso claro con las definiciones propuestas por el movimiento del acceso abierto, pues no suele especificarse la licencia de uso de ese artículo, es decir, si podemos imprimirlo, redistribuirlo o enlazarlo.

En esta línea de demarcación entre la publicación en acceso abierto y los sistemas de revisión previa, se sitúa las propuestas de self-archiving ${ }^{14}$ (Harnad, 2001). La idea básica de estas prácticas consiste en que las agrupaciones, organizaciones o instituciones interesadas en la difusión de la investigación pueden ahora aprovechar las posibilidades de las tecnologías computacionales para construir por sí mismas sistemas de archivo digital para el almacenamiento y consulta de las publicaciones sobre un determinado campo de investigación. Actualmente, muchas instituciones académicas están recogiendo esta idea para crear repositorios institucionales con los textos de los investigadores adscritos a las mismas como modo de divulgar y promocionar las líneas de investigación que financian ${ }^{15}$. En estos archivos, al igual que en las páginas personales de los investigadores, puede contener las versiones en borrador (preprint), las copias idénticas a las publicadas (postprint), o las versiones finales -corregidas y revisadas- de los artículos pero en un formato diferente al de la revista en el que fueron publicados (e-print). 
Estas dos metodologías son las que ofrecen un mayor número de artículos científicos pero también son las que, a su vez, carecen de una postura clara sobre los sistemas de revisión. De hecho, eluden explícitamente cualquier tipo de compromiso en este sentido ${ }^{16}$, razón por la que el movimiento de acceso abierto aparezca a los ojos de muchos científicos como una posibilidad de escasa fiabilidad científica, es decir, epistémicamente poco valiosa. No obstante, si bien en estos dos casos no hay una metodología específica de revisión, lo cierto es que muchos de los artículos que encontramos disponibles de esta manera son artículos ya publicados, y, por tanto, revisados, en revistas con sistema de revisión por iguales. Este sistema exige al lector, por tanto, una comprobación previa del tipo de publicación -preprint, e-print o post-print- para poder dilucidar si el artículo ha sido revisado o no.

Algunas propuestas del movimiento self-archiving muestran que, gracias a las tecnologías computacionales, no es necesaria una organización previa para iniciar el trabajo de revisión y contrastación científica. El mejor y más veterano ejemplo en este sentido es el repositorio de artículos $\operatorname{ArXiv}^{17}$. En esta ubicación electrónica los autores envían sus trabajos (aún en sus versiones preliminares) para que sean registrados y archivados electrónicamente. No existe ningún tipo de preselección ni revisión previa. Sin embargo, existen numerosos foros paralelos para el debate en los que se dirimen las controversias suscitadas por cada artículo. Controversias que, generalmente, contribuyen a la revisión del mismo y a la publicación posterior de versiones corregidas en el mismo servidor. La Red se inserta así en el proceso de puesta en público de las ideas posibilitando la creación de foros de comentaristas y correctores que ayudan a perfilar las versiones definitivas. En este caso, hay un proceso de revisión continua que produce nuevas versiones mejoradas. Proceso que, sin duda, redunda en la calidad y confiabilidad de los artículos que son sometidos, una y otra vez, a una exhaustiva revisión por toda la comunidad participante en el foro.

El ejemplo de ArXiv apunta hacia nuevas vías de organizar metodologías de revisión abiertas a través de Internet que podrian significar una serie de innegables ventajas epistemológicas. Dichas ventajas podrían servir para justificar y promocionar la extensión de la publicación de los artículos con una política de acceso abierto. A continuación se expondrán las posibilidades y desafíos de un modelo de este tipo.

\section{Revisión de artículos en la Red: POSIBILIDADES Y DESAFÍOS}

En las nuevas condiciones del entorno digital, las revistas científicas de acceso de pago justifican su existencia en función de su papel en las tareas de difusión de la ciencia y, sobre todo, en la organización de las labores de revisión previa de los artículos científicos. Cada revista coordina las comunicaciones entre los autores y un grupo de especialistas reconocidos en su materia. Estos especialistas se encargan de la evaluación y acreditación del contenido de los artículos en función de razones y criterios epistémicos $y$, a su vez, acreditan la calidad de la revista por su prestigio en la comunidad científica.

Esta organización de la revisión por los iguales en las revistas comerciales no está exenta de problemas y dificultades. La presión por conseguir publicar un artículo en las revistas de mayor impacto, las más reconocidas tanto institucional como mediáticamente, tiene desagradables efectos secundarios. El fraude es el peor de ellos y significa un verdadero problema para los sistemas de revisión actuales. Puesto que el número de revisores es limitado, su capacidad de revisión no puede detectar fraudes bien elaborados. Un ejemplo es el reciente caso del falseo de los datos sobre los experimentos de clonación del Dr. Hwang ampliamente comentado en (Semir y Revuelta, 2006). El artículo pasó todas las revisiones de los editores y sus revisores pero el fraude sólo pudo ser desenmascarado después de su publicación, es decir, cuando pudo ser contrastado por toda la comunidad (al menos, por los miembros de la comunidad que tenían acceso a la costosa suscripción) ${ }^{18}$.

Por otro lado, la competición para que un trabajo científico sea seleccionado obliga a establecer sistemas que garanticen la máxima ecuanimidad posible por parte de los revisores. Por ello, cada vez está más extendida la práctica de la revisión anónima (Blind Peer Review) que obliga a los revisores a verificar trabajos sin conocer el perfil académico de quien presentó el artículo (lo que significa un verdadero problema en ámbitos interdisciplinarios), sin conocer ni poder consultar las referencias que han sido suprimidas para garantizar el anonimato o sin poder establecer una discusión ágil y directa con el revisado. Todos estos pequeños problemas minimizan las posibilidades de generación y transmisión de conocimiento de la revisión por los iguales, así como la propia transparencia 
del sistema de revisión. La evaluación de propuestas revolucionarias, es decir, que escapan en cierta medida de los parámetros consensuados en una disciplina, la evaluación de proyectos internacionales $y$, sobre todo, las dificultades que presentan las evaluaciones en campos interdisciplinares y multidisciplinares suponen otras deficiencias del modelo (Fuller, 2002, 234).

Quizá todos estos problemas provienen de que deseamos que el sistema de revisión por los iguales cumpla con demasiadas funciones a la vez ${ }^{19}$, lo que quizá le resta efectividad en sus funciones puramente epistémicas, es decir, las de contribuir a la producción de conocimiento fiable. Un primer paso para la mejora de los sistemas de revisión en la Red puede ser tratar de separar estas funciones. De un lado, habría que implementar mecanismos relacionados con la producción de conocimiento fiable, es decir, sistemas de confirmación del conocimiento (Glymour, 1980), de colaboración y aprendizaje mutuo a través de los comentarios, de control de calidad y relevancia, de búsqueda y promoción del consenso entre científicos, etc. $Y$ por otro lado, habría que ajustar prácticas de revisión orientadas a la selección de trabajos científicos en marcos de competición y recompensa, es decir, asignación de fondos para la investigación, provisión de posiciones académicas, difusión de las investigaciones en medios de comunicación, etcétera. Se propone, por tanto, distinguir entre las labores de revisión (del conocimiento) y las de selección (de artículos o proyectos con contenidos ya revisados y confirmados previamente). Dicho en otros términos, separar la labor de los revisores como testigos de la precisión, veracidad y fiabilidad, de su labor como jurados que deben llevar a cabo selecciones y clasificaciones en ámbitos competitivos.

La propuesta de la revisión abierta se situaría en el primer caso. Todo artículo remitido sería puesto a disposición de la comunidad científica interesada para la revisión y confirmación de sus resultados. En esta línea trabajan algunas propuestas para el diseño de herramientas de publicación y revisión colectivas en Internet. Por ejemplo, en (Sumner y Buckingham Shum, 1998; Sumner, Buckingham Shum, Wright et al., 2000) se nos presenta un modelo de revisión de los textos científicos mediante una aplicación informática. En este proyecto, denominado JIME20 (Interactive Journal for Interactive Media), los textos son introducidos en una plataforma informática accesible a través de Internet. Al lado de cada texto, se integran las diversas revisiones que se han efectuado sobre el mismo, de modo que la lectura del texto se puede llevar a cabo a la vez que la de los comentarios que ha suscitado. Si la plataforma se abre a los comentarios, revisiones y posteriores contestaciones de los autores, se implementa un proceso mucho más interactivo y dinámico de producción y revisión. Los artículos pueden ser revisados una y otra vez por los autores produciendo versiones sucesivas hasta alcanzar un alto grado de consenso con la comunidad interlocutora.

En este tipo de metodologías se funden el proceso de revisión por pares (peer review) con el de los comentarios de los investigadores interesados (peer commentary). No hay separación entre el proceso de evaluación científica por toda la comunidad y el de selección previa, con lo que el segundo se ve enriquecido por el primero. Es posible así llevar a cabo un proceso de revisión más exhaustivo, evitando los errores que pueden escapar a la mirada de unos pocos revisores, por muy expertos que sean. Es de destacar que, en este sistema, el consenso se define en función de los argumentos y discusiones planteados por los revisores y comentaristas y que éstos también están disponibles en línea, enriqueciendo el propio proceso de producción. Esto constituye una ventaja sobre muchos de los actuales sistemas de revisión anónima que consisten en otorgar una determinada calificación a cada artículo y en los que se excluyen las discusiones argumentadas entre revisores y autores.

Desde el punto de vista de la epistemología social, la ventaja más importante de la revisión abierta frente a la revisión limitada a unos pocos especialistas es la mejora de la fiabilidad del proceso de evaluación por el incremento del número de revisores (Goldman, 1999, 222). Además, la apertura garantiza que puedan ser usados múltiples criterios y mecanismos de evaluación. La calidad de la revisión también depende del grado de conocimiento de los revisores. En ese sentido, sería necesario articular sistemas de recompensa académica en los procesos de revisión para lograr que un gran número de expertos se involucrase en ellos. Finalmente, la transparencia de todo el proceso y la posibilidad de ser consultado por cualquiera, y en cualquier momento, aportaría ventajas democráticas a las discusiones científicas. En definitiva, se propone el paso de un sistema en el que prima la selección opaca y la publicación de documentos estáticos, a otro que se base en la elaboración de un discurso continuo, participativo 
y abierto como primer paso y función de la revisión del conocimiento científico.

El resto de funciones de los sistemas de revisión por los iguales se podrían llevar a cabo a partir de este modelo de revisión, pero en procesos separados. En cuanto a la selección, de hecho, hay una primera selección "natural" que lleva a cabo toda la comunidad en función de las controversias, comentarios y discusiones desencadenados por cada artículo ${ }^{21}$. Se pueden cuantificar estas aportaciones, contestaciones y correcciones del documento, cuantificación que, incluso, podría ser automatizada. Este tipo de valoración cuantitativa se llevaría a cabo sobre la base de criterios e índices bastante similares a los que existen en los sistemas actuales de valoración de la relevancia de los artículos científicos. La ventaja para los científicos es que esa valoración sería independiente, en principio, de la importancia de la publicación o institución en la que se haya conseguido publicar el artículo. Si una revista o una institución pretenden seleccionar artículos para la publicación o temas relevantes y "candentes" para la financiación de proyectos de investigación sobre los mismos, un primer dato a tener en cuenta puede ser el relacionado con este trabajo de poner a disposición de la comunidad el conocimiento y ajustarlo hasta lograr un consenso. Otras funciones que necesitan el anonimato o la confidencialidad, como la asignación de fondos para proyectos de investigación, quedarían fuera de un sistema con esta transparencia, a no ser por la evaluación de la actividad académica anterior de los científicos.

La capacidad de un sistema de revisión abierta para eliminar problemas como el nepotismo, las malas prácticas investigadoras, la creación y retroalimentación de grupos o temáticas académicas interesadas, etc. es, en realidad, bastante pequeña. Todos esos fenómenos pueden reproducirse, incluso agravarse, con sistemas de revisión abierta. Sin embargo, la transparencia inherente al sistema proporcionaría las herramientas necesarias para detectar (aun cuando no corregir) estos problemas. Obligaría a los científicos a aplicar su espíritu crítico también a sus colegas, sus prácticas y sus instituciones. Además, transformaría el nepotismo en un ejercicio productivo, hasta cierto punto, pues para "echar una mano a un amigo" habría que publicar un comentario sobre el artículo a favorecer que, al estar abierto a toda la comunidad, debería tener contenido e interés investigador. Pero, puesto que esas prácticas se desarrollan en el ámbito de la competición científica, en la medida en que la revisión abierta vaya convirtiéndose en la herramienta de comunicación, intercambio y producción colaborativa más importante de los investigadores, esos problemas seguramente se trasladarían a otros ámbitos en los que sí pueda establecerse la competición.

Por otro lado, los errores son, si acaso, similares a los del sistema editorial actual. Puede darse el caso de que artículos poco relevantes sean muy comentados $y$, por tanto, valorados en función de criterios cuantitativos, y que artículos muy interesantes o revolucionarios no desencadenen ningún tipo de debate o diálogo.

Para finalizar, conviene analizar cómo se podrían dividir y organizar todas estas funciones epistemológicas entre las diversas propuestas de acceso abierto y los modelos de negocio editorial existente. Un sistema de revisión abierta mediado por tecnologías computacionales como el propuesto sólo puede llevarse a cabo si se puede acceder libremente a los artículos. En este sentido, el acceso abierto es una condición necesaria para implementar la ventaja epistemológica fundamental de las publicaciones científicas en línea, es decir, la posibilidad de producir y difundir conocimiento científico más fiable y veraz. Para ello, las actuales iniciativas institucionales para el autoarchivado en acceso abierto de los documentos de sus investigadores podrían perfectamente ampliarse con espacios electrónicos para los comentarios, las revisiones y las discusiones directamente vinculadas con cada artículo. Universidades, asociaciones de investigadores sin ánimo de lucro o fundaciones para la promoción del conocimiento podrian ser los mejores candidatos para encargarse de estas labores, siempre que dichas instituciones las organizasen con total transparencia, sin ánimo de lucro y respetando las condiciones de acceso abierto a lo publicado.

Por su parte, las revistas de investigación, con vocación comercial o sin ella, podrían ofrecer servicios de selección temática, elaboración de monografías o propuestas editoriales para investigar sobre nuevos campos. Se convertirian así en catalizadores de discusiones académicas en línea o podrian centrar el otro grupo de labores epistémicas propuesto, es decir, la selección de trabajos o líneas de investigación. La enorme cantidad de información generada por un sistema de acceso y revisión abierta otorgaría una mayor importancia a estas labores de selección, así 
como al trabajo de los expertos que las llevasen a cabo. El modelo de negocio se podría mantener en función de este servicio adicional de selección, organización y edición cuidada de artículos, revisiones y comentarios. En esta línea, las revistas pueden jugar un importante papel para seleccionar y promocionar tendencias o líneas novedosas de investigación usando su capacidad para la difusión y el impacto mediático. Tanto el negocio basado en la selección, como el basado en la difusión o promoción conciliarian estas labores editoriales con los argumentos morales y económicos sobre el acceso abierto, pues siempre habría otro camino para obtener los textos de manera gratuita y, sobre todo, para consultar todo el conocimiento relacionado con su producción, independientemente de las selecciones realizadas.

\section{Conclusión}

Se ha propuesto un modelo de publicación y revisión de los artículos científicos que concilie los beneficios económicos y morales de las publicaciones en acceso abierto a través de Internet y las posibilidades para la mejora de las prácticas de producción, difusión y validación del conocimiento de este entorno tecnológico. Desde este punto de vista epistemológico, las posibilidades del acceso abierto parecen también incuestionables pues son inherentes a la propia empresa colaborativa de construcción del conocimiento científico, técnico y humanístico. Sin embargo, esta afirmación choca con descripciones pragmáticas de la actividad de los científicos, aparentemente cada vez más dominada por una cultura de la competición. Por ello, el desafío más importante al que se enfrentan las publicaciones de acceso abierto es el de ofrecer sistemas que, poco a poco, vayan venciendo las inercias de los sistemas de publicación que, durante años, se han constituido en la base de la evaluación de la actividad investigadora. Si la competición entre científicos ha de existir, ha de establecerse sobre la base de un conocimiento confirmado, fiable y relevante, es decir, debe establecerse después de la elaboración y contrastación colaborativa y plural del conocimiento. Estas prácticas metodológicas no pueden estar sometidas a criterios competitivos porque, en ese caso, se abren vías para el fraude, el robo de ideas y la manipulación de las líneas de investigación en función de intereses no epistémicos. La competición que se plantea en los actuales sistemas de publicación de los resultados científicos para conseguir un espacio en una de las revistas de mayor factor de impacto ha contribuido a abrir alguna de estas vías al mezclar las labores de contrastación y confirmación con las labores de selección editorial.

El modelo propuesto debe comenzar por una revolución en los sistemas de revisión de la literatura científica, mediante la posibilidad que ofrecen las tecnologías computacionales para el despliegue de prácticas de elaboración y contrastación abierta, dinámica y plural de la información y el conocimiento científico en Internet. Para ello, la reflexión epistemológica debe distinguir entre los sistemas de revisión de los trabajos científicos para confirmar y validar el conocimiento que atesoran y los sistemas de selección de trabajos científicos en un marco de escasez de los recursos, económicos o editoriales. El sistema de revisión abierta (open peer review) puede desempeñar las funciones del primer sistema combinando las posibilidades de producción y publicación en formato digital, con las posibilidades de comunicación horizontal a través de Internet. Obtendriamos así un mecanismo de publicación e interlocución ilimitada entre los científicos con interesantes ventajas epistemológicas.

Si queremos hablar de Sociedad del Conocimiento, no sólo debemos preocuparnos de garantizar el acceso a la información, en este caso científica, sino a los procedimientos y metodologías mediante los que ese conocimiento es discutido y validado. Las posibilidades tecnológicas y epistemológicas para desarrollar sistemas que, como el propuesto, ofrezcan garantías mínimas de transparencia y democracia en dichos procedimientos, pueden suponer un primer paso para reducir la desconfianza de la ciudadanía en los resultados de la investigación científica y tecnológica. 
1 La redacción de este trabajo se ha beneficiado de la financiación del proyecto investigación del Ministerio de Ciencia y Tecnología HUM200502105/FISO. Agradezco a Karim Gherab sus comentarios y valiosas aportaciones durante el proceso de revisión de este artículo.

๑ 2007 Roberto Feltrero, <http://www. uned.es/dpto_log/rfeltrero>. Algunos derechos reservados. Se permiten la copia, distribución, uso y comunicación de esta obra si se respetan las siguientes condiciones:

- Se debe reconocer explícitamente la autoria de la obra incluyendo esta nota y su enlace.

- La copia será literal y completa.

- No se podrá hacer uso de los derechos permitidos con fines comerciales, salvo permiso expreso del autor.

El texto precedente es una nota orientativa de la licencia bajo la que se distribuye este documento cuyo texto completo puede consultarse en: <http://creativecommons.org/licenses/by-nc-nd/2.0/deed.es>

2 Algunos artículos clásicos que resumen las propuestas del acceso abierto son (Harnad, 1995, 1996). Para una revisión completa de toda la temática ver (Willinsky, 2006)

3 Es interesante destacar la recomendación explícita sobre el acceso abierto a los artículos y resultados científicos provenientes de proyectos de investigación financiados públicamente que se hace desde el Consejo de Europa, más concretamente en la sesión n. 2832 del Consejo de Competitividad que se recoge en el comunicado de prensa 14865/07 (pp. 37-44) disponible en <http://www.consilium. europa.eu/ueDocs/cms_Data/docs/ pressdata/es/ecofin/97943.pdf>.
4 Aunque poco a poco algunas revistas de acceso abierto empiezan a ganar posiciones en esta clasificación $<$ http://scientific.thomson.com/media/presentrep/essayspdf/openaccesscitations2.pdf>.

5 El concepto de tecnologías computacionales abarca todos los artefactos tecnológicos capaces de llevar a cabo el tratamiento automático de información en formato digital mediante procesos de cómputo controlados por software. Para una caracterización completa de este concepto, consultar (Feltrero, 2006).

6 En Internet, el más conocido es Google Scholar, pero todos los grupos editoriales tienen el suyo propio de igual manera que la mayoría de las universidades o instituciones han diseñado uno para búsquedas en las bases de datos bibliográficas y de contenidos a las que están suscritas.

7 Cuando las aplicaciones proporcionan herramientas para que los autores editen y publiquen por sí mismos sus artículos (como es el caso de ArXiv $<$ http://es.arxiv.org/> o de, por ejemplo, el archivo de acceso abierto de la UNED <http://62.204.194.47/fez/>) dichos costes se reducen al mantenimiento del servidor y la aplicación informática. Estos ejemplos ponen en evidencia las deficiencias de algunos argumentos (Esposito, 2004) que apuntan a los altos costes que siguen suponiendo estas actividades en el nuevo entorno tecnológico.

8 Una introducción histórica muy interesante sobre los detonantes de movimiento de acceso abierto, se puede consultar en el artículo de Jean Claude Guédon "In Oldenburg's Long Shadow: Librarians, Research Scientists, Publishers, and the Control of Scientific Publishing", disponible en http://eprints.rclis.org/
Aceptado: 28 de diciembre de 2007 
archive/00003951/01/ARL_Proceedings_138_In_Oldenburg's_Long_ Shadow,_by_Guedon.htm.

9 En los primeros capítulos (Willins$k y, 2006)$ se pueden encontrar estos argumentos morales expuestos con gran profusión de ejemplos.

10 Ése es el denominador común de las propuestas recogidas en las definiciones elaboradas en la Budapest Open Access Initiative <http://www. soros.org/openaccess/read.shtml> de febrero de 2002 o en declaración de 2003 en Berlín sobre el acceso abierto al Conocimiento en las Ciencias y las Humanidades <http://www.zim. mpg.de/openaccess-berlin/berlindeclaration.html>.

11 En <http://www.doaj.org/home> se puede encontrar una lista completa de las revistas que practican la política del acceso abierto.

12 Por ejemplo, la popular Public Library of Science exige en algunos casos el pago de hasta 1250 USD a los autores <http://www.plosone. org/static/policies.action>. Aunque también se ofrecen exenciones de estas cuotas a los autores que demuestren su falta de recursos económicos.

13 Un buen ejemplo de cómo se ha de llevar a cabo esta práctica es la página web del filósofo Luciano Floridi <http://www.wolfson.ox.ac.uk/ floridi/> pues en sus documentos se encuentran las correspondientes notas aclaratorias de la condición de borrador de los artículos alli presentados y las restricciones concretas que se imponen sobre el uso de esos preprints.

14 Algunos ejemplos de sistemas de archivo digital son ArXiv <http:// es.arxiv.org/>, E-prints <http://www. eprints.org/> o Cogprints <http://cogprints.org/>.
15 Ejemplos de este tipo de repositorios pueden encontrar en $<$ http://62.204.194.47/fez/> o en http://www.madrimasd.org/informacionidi/e-ciencia/buscar-documentos/default.asp. En el sitio de Internet <http://www.sherpa.ac.uk> ofrece todo tipo de información y enlaces sobre estas iniciativas institucionales.

16 Ver el apartado "What Open Access is not" en <http://www.sherpa.ac.uk/ guidance/authors.html>.

17 http://es.arxiv.org/.

18 Los fallos de los sistemas de revisión también se producen en el campo de las humanidades. Una buena prueba es el famoso caso Sokal (se puede consultar un breve resumen del mismo y la bibliografía asociada en <http://en.wikipedia.org/wiki/ Sokal_Affair $>$ ).

19 En (Fuller, 2002, 233) podemos encontrar un precisa enumeración de todas ellas.

20 La página general del proyecto es $<$ http://www-jime.open.ac.uk> y en esta otra <http://www-jime.open. ac.uk/about.html\#lifecycle> se puede encontrar una interesante propuesta de este grupo para dividir y engarzar los diversos pasos de la revisión según su propio modelo epistemológico.

21 Incluso existe una "autoselección" previa (self-selection bias) de los repositorios digitales, pues el propio autor preselecciona entre sus artículos aquellos que considera mejores para publicar en la Web (ver Kurtz y Brody, 2006, 49).

\section{BIBLIOGRAFÍA}

Dominy, P. y Bhatt, J. (2006): Peer Review in the Google Age. Is technology chan- ging the way science is done and evaluated?, disponible en junio 2006.

Esposito, J. J. (2004): "The devil you don't know: The unexpected future of Open Access publishing", First Monday, 9 (8), http://firstmonday.org/issues/issue9_8/esposito/index.html.

Feltrero, R. (2006): "Ética de la Computación: Principios de Funcionalidad y Diseño", Isegoría, 34, pp. 79-109.

Feltrero, R. (2007): "Tecnologías Cognitivas, ¿Metodologías Convergentes?", Anthropos, 214, pp. 96-106.

Fuller, S. (2002): Knowledge Management Foundations, Boston-0xford, Butterworth Heinemann.

Glymour, C. (1980): Theory and Evidence, Princeton, Princeton U. Press.

Goldman, A. (1999): Knowledge in a Social World, Oxford, Clarendon Press.

Goldman, A. (2002): Pathways to Knowledge, New York, Oxford Univ. Press.

Harnad, S. (1995): "Sorting the esoterica from the exoterica: there is plenty of room in Cyberspace: Response to Fuller", Information Society, 11 (4), pp. 305-324, http://cogprints.soton. ac.uk/documents/disk0/00/00/16/84/ index.html.

Harnad, S. (1996): "Implementing Peer Review on the Net: Scientific Quality Control in Scholarly Electronic Journals", en R. Peek y G. Newby (eds.), Scholarly Publishing: The Electronic Frontier, Cambridge MA, MIT Press, http://cogprints.soton.ac.uk/documents/disk0/00/00/16/92/index.html.

Harnad, S. (1998): "Learned inquiry and the net: The role of peer review, peer commentary and copyright", Antiquity, 71, pp. 1042-1048.

Harnad, S. (2001): "The self-archiving initiative", Nature, 410, pp. 1024-1025, http://www.ecs.soton.ac.uk/ harnad/ Tp/nature4.htm.

Harnad, S. y Brody, T. (2004): "Comparing the impact of Open Access (OA) vs. 
Non-OA articles in the same journals", D-Lib Magazine, 10 (6).

Kurtz, M. y Brody, T. (2006): "The impact loss to authors and research", en Neil Jacobs (ed.) (2006): Open Access: Key Strategic, Technical and Economic Aspects, Chandos Publishing.

Lipinski, T. A. y Britz, J. J. (2000): "Rethinking the ownership of information in the 21st century: Ethical implications", Ethics and information technology, 2 (1), pp. 49-71.

Longino, H. (1990): Science as Social Knowledge, Princeton, Princeton Univ. Press.
Pringle, J. (2004): "Do open access journals have impact?", Nature (Web Focus) http://www.nature.com/nature/focus/ accessdebate/19.html.

Semir, V. y Revuelta, G. (2006): "Dr. Hwang and the clone that never was", Quark, 37-38, pp. 105-123.

Stallman, R. (2004): Software Libre para una Sociedad Libre, Madrid, Traficantes de Sueños.

Sumner, T. y Buckingham Shum, S. (1998): "From Documents to Discourse: Shifting Conceptions of Scholarly Publishing", Proc. CHI 98: Human Factors in
Computing Systems, Los Ángeles, CA, ACM Press, NY, pp. 95-102.

Sumner, T.; Buckingham Shum, S.; Wright, M. y Bonnardel, N. et al. (2000): Redesigning the Peer Review Process: A Developmental Theory-in-Action, Ponencia presentada en: COOP'2000: Fourth International Conference on the Design of Cooperative Systems, Sophia Antipolis, France.

Willinsky, J. (2006): The access principle: the case for open access to research and scholarship, Cambridge, Mass., MIT. 